\title{
Indicadores de resíduos de construção e demolição para construções residenciais novas
}

\author{
Construction and demolition waste indicators for new \\ residential buildings
}

\section{Miguel Mália \\ J orge de Brito Miguel Bravo}

\section{Resumo}

A

indústria da construção representa um dos maiores e mais activos sectores da União Europeia (UE), consumindo mais matérias-primas e energia do que qualquer outra actividade económica. Da mesma forma, os resíduos das suas actividades corespondem a uma elevada parcela dos resíduos produzidos em toda a UE. A actual legislação na UE visa criar condições para a implementação de medidas de prevenção e reciclagem de RCD. No entanto, carece de instrumentos que acelerem o desenvolvimento de um sector tão tradicional como o da construção. O presente trabalho tem por objectivo determinar indicadores que possibilitem estimar a quantidade de RCD gerados, ao nível da obra, tanto globalmente como por fluxo de resíduos. A geração de RCD foi estimada para seis sectores específicos: nova construção residencial, nova construção não residencial, demolição residencial, demolição não residencial, reabilitação residencial e reabilitação não residencial. Neste artigo, apresentam-se os resultados para a nova construção residencial. Os dados necessários ao

Miguel Mália

Departamento de Engenharia Civil, Arquitectura e Georrecursos

Universidade Técnica de Lisboa Avenida Rovisco Pais, 1 1049-001 Lisboa - Portugal Te.: +351 (91) 356-0132 E-mail: miguel_malia@hotmail.com

J orge de Brito Departamento de Engenharia Civil, Arquitectura e Georrecursos Universidade Técnica de Lisboa Tel.: +351 (21) 841-9709 E-mail: jb@civil.ist.utl.pt

Miguel Bravo Departamento de Engenharia Civil, Arquitectura e Georrecursos Universidade Técnica de Lisboa Tel.: +351 (21) 841-8431 E-mail: miguelnbravo@gmail.com

Recebido em 19/05/11 Aceito em 30/08/11 desenvolvimento dos indicadores foram obtidos através de um levantamento exaustivo dos dados de estudos anteriores.

Palavras-chave: Resíduos de construção e demolição. Legislação. Quantificação. Indicadores.

\section{Abstract}

The construction industry is one of the largest and most active sectors of the European Union (EU), consuming more raw materials and energy than any other economic activity. Likewise waste from its activities corresponds to a large share of those produced in the EU. The present EU legislation aims at creating conditions to implement construction and demolition waste $(C D W)$ prevention and recycling measures. However, there is a lack of tools to accelerate the development of a sector as traditional as construction. The main objective of this research work is to propose indicators for estimating the amount of CDW generated on site, both globally and by waste stream. CDW generation was estimated for six specific sectors: new residential construction, new nonresidential construction, residential demolition, non-residential demolition, residential rehabilitation, and non-residential rehabilitation. This paper presents the results for new residential construction. The data used to develop the indicators was obtained through an extensive analysis of data from previous international studies.

Keywords: Construction and demolition waste. Legislation. Quantification. Indicators. 


\section{Introdução}

A indústria da construção constitui um dos maiores e mais ativos setores em toda a Europa, representando $28,5 \%$ e $7,0 \%$ do emprego, respectivamente, na indústria e em toda a economia europeia (EUROSTAT..., 2010). O peso dessa atividade reflete-se também no nível ambiental. O setor consome mais matérias-primas e energia do que qualquer outra atividade econômica e produz a maioria dos resíduos gerados em todo o espaço comunitário.

Relativamente aos resíduos, essa indústria gera mais de 500 milhões de toneladas por ano de resíduos por toda a UE. Esse fluxo de resíduos representa cerca de $25 \%$ a $30 \%$ de todos os resíduos produzidos (EUROSTAT..., 2010). Esses resíduos, denominados de resíduos de construção e demolição (RCD), resultam da atividade de nova construção, reabilitação e demolição de edifícios, obras públicas e outras estruturas. Por sua vez, no Brasil a geração de RCD per capita foi estimada em 500 kg/habitante.ano (PINTO, 1999). Segundo o mesmo autor, esses resíduos representam entre $41 \%$ e $70 \%$ do total dos resíduos sólidos urbanos, nos centros urbanos do Brasil.

Os RCD têm uma constituição heterogênea, sendo compostos de frações de diversas dimensões. A fração com maior volume é a dos materiais inertes, variando entre $40 \%$ e $85 \%$ do volume total de resíduos, quando não são contabilizados os solos de escavação (EUROSTAT..., 2010). O betão e os materiais cerâmicos são a principal fonte de material inerte, como se mostra no Quadro 1.

Devido à natureza inerte dos RCD, não lhes é dada tanta importância, apesar de representarem grandes volumes, como acontece no caso dos resíduos sólidos urbanos (COCHRAN et al., 2007; LAGE et al., 2010). No entanto, nos últimos anos, tem sido dada mais atenção a esse fluxo de resíduos, devido ao surgimento de questões relacionadas com eventuais impactos ambientais e com o potencial de reciclagem de componentes dos RCD
(BERGSDAL; BOHNE; BRATTEBØ, 2007; COCHRAN et al., 2007).

Apesar de os RCD terem alto potencial de recuperação, apenas uma pequena parte é realmente recuperada (BERGSDAL; BOHNE; BRATTEBØ, 2007; ORTIZ; PASQUALINO; CASTELLS, 2010). Atualmente, 75\% dos RCD produzidos na UE são depositados em aterro (ORTIZ; PASQUALINO; CASTELLS, 2010). No entanto, já foram atingidas taxas de reaproveitamento superiores a $80 \%$ em países como a Holanda, Dinamarca e Alemanha (EUROSTAT..., 2010).

A fim de preservar o meio ambiente e garantir a correta gestão dos RCD, uma grande variedade de regulamentações ambientais tem sido estabelecida. A maioria dessas leis procura minimizar e controlar os RCD (SOLÍS-GUZMÁN et al., 2009; ORTIZ; PASQUALINO; CASTELLS, 2010). No entanto, é difícil cumprir e fazer cumprir a legislação existente quando não existem ferramentas que permitam controlar a quantidade de RCD produzidos. O primeiro passo para a correta gestão desse tipo de resíduos passa por determinar sua real quantidade (LAGE et al., 2010).

Na primeira parte deste trabalho, é realizada uma pesquisa do panorama atual da legislação sobre RCD na UE, em alguns países da comunidade e no Brasil. Esta pesquisa visa mostrar a necessidade de essas leis serem complementadas com instrumentos que permitam estimar a quantidade de RCD produzida no local da obra. Posteriormente, este estudo visa determinar indicadores que forneçam à indústria de construção um conjunto de valores confiáveis que possam ser utilizados como referência para quantificar os RCD gerados ao nível da obra. Esses indicadores foram determinados mediante um levantamento exaustivo dos dados dos estudos existentes.

\begin{tabular}{l|c|c|c|c}
\hline \multirow{2}{*}{ Material } & & \multicolumn{2}{|c}{ Composição dos RCD (\%) } & $\begin{array}{c}\text { Bergsdal, } \\
\text { Bohne e } \\
\end{array}$ \\
& Pereira, 2002 & Costa e Ursella, 2003 & $\begin{array}{c}\text { Maña I Reixach, Barroso e } \\
\text { Cusco, 2000 } \\
\text { Brattebo, } \\
\mathbf{2 0 0 7}\end{array}$ \\
\hline $\begin{array}{l}\text { Betão, alvenaria e } \\
\text { argamassa }\end{array}$ & 58,3 & 84,3 & 85,0 & 67,24 \\
Metais & 8,3 & 0,08 & 1,8 & 3,63 \\
Madeira & 8,3 & - & 11,2 & 14,58 \\
Plásticos & 0,83 & - & 0,20 & - \\
Asfalto & 10,0 & 6,9 & - & - \\
Outros & 14,2 & 8,8 & 1,8 & 14,55 \\
\hline \multicolumn{1}{c|}{ Total } & $\mathbf{1 0 0}$ & $\mathbf{1 0 0}$ & $\mathbf{1 0 0}$ & $\mathbf{1 0 0}$ \\
\hline
\end{tabular}

118 Mália, M.; Brito, J. de; Bravo, M. 
Quadro 1 - Composição dos RCD com base em alguns estudos publicados

Fonte: Mália (2010).

\section{Legislação e estratégias para os RCD}

Com elevada frequência, assiste-se à deposição em aterro dos RCD, sem nenhum controle e sem qualquer preocupação de separação na origem. Para agravar a situação, verifica-se também um número significativo de despejos ilegais com consequências graves não só para o ambiente mas também para a saúde pública. De modo a resguardar o ambiente e a garantir o crescimento sustentável das cidades, um grande número de códigos ambientais e iniciativas tem vindo a ser implementado. Para além de fiscalizar, a gestão de RCD visa encontrar soluções que conduzam à valorização de RCD através de normas e especificações técnicas que credibilizem seu uso como material reciclado e reutilizável.

Na União Europeia, ainda não existe legislação específica para os RCD, como se verifica para outros tipos de resíduos. No entanto, em 1975, surgiu a primeira legislação europeia com regulamentação dos resíduos em geral (Diretiva 75/442/CEE). Esta tinha como principais objetivos garantir uma eliminação de resíduos que colocassem em risco a saúde humana e o ambiente, e incentivar a recuperação de resíduos e sua reutilização, a fim de preservar os recursos naturais. Em 1991, a política da União Europeia modifica-se e surge a Diretiva 91/156/CEE. Para além dos objetivos referidos quando da anterior diretiva, esta visava principalmente assegurar que os diversos países da União Europeia se tornavam autossuficientes no que se refere à eliminação de resíduos. Por fim, em 2008, foi publicada a Diretiva 2008/98/EC, que foca os seguintes aspectos:

(a) minimizar o impacto negativo da produção e gestão de resíduos na saúde humana e no ambiente; harmonizar a legislação em âmbito europeu, clarificando não só as definições usadas mas também a distinção entre valorização e eliminação, e resíduo e não resíduo; reduzir a utilização de recursos e propiciar a aplicação prática da hierarquia de gestão de resíduos; e

(b) garantir que a redução de resíduos constitui a primeira prioridade e que a reutilização e reciclagem têm prioridade em relação à valorização energética dos resíduos.

Essa diretiva marca a transição de uma política que se baseava na eliminação de resíduos para uma que privilegia a utilização desses resíduos através de sua reciclagem, de modo a poupar os recursos naturais.
Apesar de a Diretiva 2008/98/EC não ser específica para nenhum tipo de resíduo, nesta existem duas importantes referências aos RCD. Na primeira, encontra-se uma solução para os solos de escavações, que representam a maior fracção dos RCD e que, em alguns casos, como descreve a diretiva, não deveriam sequer ser considerados como resíduos. Na segunda referência, são traçadas metas para a reciclagem de RCD na UE.

Apesar de não existirem leis comunitárias próprias, alguns países da União Europeia tomaram diversas ações. Entre estas, destacam-se as várias iniciativas tomadas para estimular a redução e o reaproveitamento de RCD. Descreve-se aqui, de um modo geral, o panorama dos RCD nos países mais desenvolvidos nessa área. Assim, optou-se por caracterizar a Dinamarca, a Alemanha e o Reino Unido. Avalia-se também a gestão de RCD na Espanha e em Portugal, países menos desenvolvidos nessa temática. Por fim, indicam-se as estratégias seguidas no Brasil.

\section{Dinamarca}

A Dinamarca é um dos maiores casos de sucesso no que diz respeito à gestão de RCD. Nesse país a reciclagem deles é uma prática corrente. O objetivo de atingir uma taxa de reciclagem de $90 \%$ em 2004 foi alcançado em 1997 e manteve-se nesse nível desde então (WASTE..., 2010). Essa elevada taxa de reciclagem deve-se a dois importantes fatores: o elevado imposto a que estão sujeitos os resíduos que não são reciclados e a obrigatoriedade de separação dos resíduos na origem.

O imposto sobre resíduos foi instituído em 1990 e revelou-se um instrumento eficaz no aumento da reciclagem de RCD. Ele levou os geradores de resíduos a reduzir as quantidades de resíduos que produzem e a reciclar os resíduos (MONTECINOS; HOLDA, 2006). A extração de agregados naturais encontra-se também sujeita ao pagamento de uma taxa específica.

Em 1995, foi publicado o regulamento municipal sobre a triagem de RCD (WASTE..., 2010). As câmaras municipais ficaram encarregadas do dever de elaborar regulamentação sobre os RCD, a fim de aumentar sua reciclagem. Essa regulamentação impunha a separação de RCD na fonte quando o total de resíduos produzidos fosse superior a uma tonelada (MONTECINOS; HOLDA, 2006). Isso significa que mesmo os edifícios de menores dimensões estão obrigados a separar os resíduos na origem. 


\section{Reino Unido}

O Reino Unido é um dos países que mais RCD produz dentro da União Europeia. No entanto, é também um daqueles que mais políticas têm implementado, de modo a reaproveitá-los.

O Environmental Protection Act, de 1990, estabelece a base para o controle da concessão de licenças e outras disposições destinadas a assegurar que o tratamento de resíduos e sua eliminação ou recuperação não prejudiquem o ambiente. Em 1996, foi implementada uma taxa para a deposição de RCD em aterro. Essa taxa sofreu anualmente diversos aumentos. Por sua vez, em 2002, foi introduzido um imposto sobre os agregados naturais, para garantir que o impacto ambiental de sua extração é plenamente refletido no preço. Esse imposto visa também estimular o uso de produtos reciclados a partir de RCD, em substituição dos produtos naturais.

Em 2008, foi publicada uma nova regulamentação. Esta torna os planos de gestão de RCD na obra obrigatórios para todos os projetos de construção que custem mais de R\$ 791.142 ( $€$ 341.880). Esse plano de gestão de RCD, Site Waste Management Plan (SWMP), registra a quantidade e o tipo de resíduos produzidos no estaleiro e a forma como os RCD vão ser reutilizados, reciclados ou eliminados.

\section{Espanha}

Na Espanha, a reciclagem de RCD ainda se encontra pouco desenvolvida, sendo a taxa de reciclagem menor do que $10 \%$. Tal como em Portugal, só em 2008 foi publicada legislação para regular a produção e gestão desse fluxo específico de resíduos.

O Real Decreto 105/2008, de 1 de fevereiro de 2008, obriga o arquiteto a incluir um estudo sobre a gestão de RCD na fase de projeto e obriga o construtor a desenvolver um plano de gestão de RCD para a obra. Tanto o estudo como o plano são necessários para a obtenção da licença de construção e devem conter dois aspectos importantes: as quantidades de resíduos e o custo do tratamento deles (SÓLIS-GUZMÁN et al., 2009). O decreto impõe também a obrigação de separação dos RCD na origem, a partir de certos limites, e proíbe a deposição de RCD sem tratamento prévio.

Para atingir níveis de reciclagem aceitáveis, as autoridades espanholas pretendem, através de regulamentos futuros, incidir na erradicação de aterros ilegais e alterar os preços de admissão de RCD em aterros legalizados. Querem também estimular a procura de produtos reciclados de
RCD, através da aplicação de normas a esses materiais que facilitem sua inclusão em obra (MINISTERIO..., 2010).

\section{Portugal}

Em Portugal, a legislação para a regulamentação do fluxo de RCD foi aprovada em 2008. Esta surgiu para combater as grandes quantidades que são produzidas desse resíduo e as frequentes deposições ilegais de RCD.

O regime jurídico de gestão de resíduos foi pela primeira vez aprovado em Portugal através do Decreto-lei $n^{\circ}$ 488/85 (LISBOA, 1985). Contudo, a evolução rápida do direito comunitário determinaria a revogação daquele decreto pelo Decreto-lei no 310/95 (LISBOA, 1995), que tratou de operar a transposição para a ordem jurídica interna das diretivas europeias. Mais tarde, este seria revogado pelo Decreto-lei $n^{\circ} \quad 239 / 97$ (LISBOA, 1997), que estabelecia as regras gerais para a correta gestão de resíduos. Atualmente, encontra-se em vigor o Decreto-lei $n^{\circ}$ 178/2006 (LISBOA, 2006). Segundo este decreto, as obrigações gerais são:

(a) separar seletivamente os resíduos na origem; enviar os resíduos para entidades licenciadas, para sua gestão;

(b) proceder ao licenciamento das operações de gestão de resíduos;

(c) cumprir as regras sobre operações de transporte de resíduos; e

(d) registrar eletronicamente o envio de resíduos.

Apesar de todo o esforço legislativo que a gestão de resíduos sofreu desde 1985, só em 2008 foi publicada a primeira regulamentação específica para gestão de RCD (LISBOA, 2008). Esse diploma estabelece o regime de operações de gestão dos RCD, definindo as operações de recolha, transporte, armazenamento, triagem, tratamento, valorização e eliminação dos RCD.

O Decreto-lei $n^{\circ}$ 46/2008 (LISBOA, 2008) exige que se estime não só a quantidade de resíduos produzidos no global da obra mas também a quantidade dividida por fluxo específico de resíduos, identificando-os segundo o código da Lista Europeia de Resíduos (LER). Assim, o objetivo principal deste trabalho foi 0 desenvolvimento de indicadores que possibilitem estimar a geração global de RCD na obra, bem como sua composição. 


\section{Brasil}

No Brasil, a Resolução do Conselho Nacional do Meio Ambiente (Conama) $\mathrm{n}^{\mathrm{o}}$ 237, de 1997 (CONSELHO..., 1997), começou por indicar que as atividades de tratamento e disposição final de resíduos sólidos deveriam estar sujeitas ao licenciamento ambiental do órgão competente (ANGULO, 2000).

Em julho de 2002, o Conama aprovou a Resolução $n^{\circ} 307$ (CONSELHO..., 2002), que leva em consideração as definições da Lei de Crimes Ambientais, de 1998. Essa lei previa penalidades para a disposição final de resíduos em desacordo com a legislação. A Resolução n ${ }^{0} 307$ estabelece diretrizes, critérios e procedimentos para a Gestão dos Resíduos da Construção Civil, criando responsabilidades para a cadeia geradortransportador-receptor-municípios.

Essa resolução exige do poder público municipal a elaboração de leis, decretos, portarias e outros instrumentos legais que resolvam os problemas oriundos do tratamento inadequado dos RCD (MINISTÉRIO..., 2005). No âmbito estadual de São Paulo, foi editada em 2002 a Resolução SMA $\mathrm{n}^{\circ} 41$, que teve como objetivo a gestão de forma organizada do destino final dos resíduos em todo o Estado. Essa resolução estabeleceu prazos para a adequação das áreas onde habitualmente se colocavam os RCD, para que esses locais fossem transformados em áreas de aterro, com as condições específicas de operação previstas nas normas técnicas já existentes.

Em 2004, a Associação Brasileira de Normas Técnicas (ABNT) publicou cinco normas relacionadas com os RCD:

(a) NBR 15112 (ABNT, 2004a): diretrizes para projeto, implantação e operação de áreas de triagem e transbordo;

(b) NBR 15113 (ABNT, 2004b): diretrizes para projeto, implantação e operação de aterros;

(c) NBR 15114 (ABNT, 2004c): diretrizes para projeto, implantação e operação de áreas de reciclagem;

(d) NBR 15115 (ABNT, 2004d): procedimentos para execução de camadas de pavimentação utilizando agregados reciclados de resíduos da construção; e

(e) NBR 15116 (ABNT, 2004e): requisitos para utilização em pavimentos e preparo de concreto sem função estrutural com agregados reciclados de resíduos da construção.

As três primeiras normas apresentam uma metodologia semelhante e pretendem controlar a implantação, projeto e operação das diversas fases da gestão dos RCD. As NBR 15115 (ABNT, 2004d) e NBR 15116 (ABNT, 2004e) estabelecem critérios para a produção de agregados reciclados com qualidade, incentivando a utilização desses materiais na forma de matéria-prima, para que deixem de causar impactos ambientais (WIENS; HAMADA, 2006).

\section{Metodologia proposta para a quantificação de RCD}

Existem muitas formas de efetuar uma estimativa dos RCD gerados em obra. Por exemplo, na Holanda, Bossink e Brouwers (1996) concluíram que a quantidade de material de construção comprado que acaba como resíduo no local de construção varia entre $1 \%$ e $10 \%$ (por peso), sendo a quantidade média de $9 \%$. Segundo Pinto e Agopyan (1994), no Brasil, a taxa de perda na atividade de construção é de $20 \%$ a $30 \%$ do peso total de materiais no estaleiro. No Reino Unido, o British Research Establishment (2010) desenvolveu um método de quantificação dos RCD produzidos ao nível da obra, o SMARTWaste ${ }^{\mathrm{TM}}$. Essa ferramenta disponibiliza valores de referência que permitem estimar a quantidade de RCD gerados em projetos de nova construção. Os dados utilizados para produzir essas estimativas são retirados de projetos inseridos no sistema SMARTWaste ${ }^{\mathrm{TM}}$. O método utilizado por Maña i Reixach, Gonzàlez i Barroso e Sagrera i Cuscó (2000), na Espanha, permite obter o volume, por unidade de área, de resíduos gerados na atividade de nova construção e demolição de edifícios. Os resíduos de construção são estimados para três fases construtivas distintas: estrutura, execução de alvenaria e acabamento. Os resíduos de demolição são calculados em função do tipo de construção: edifícios residenciais com estrutura em alvenaria ou betão armado, e edifícios industriais com estrutura em alvenaria. Para produzir esses indicadores, foram acompanhadas várias obras na região da Catalunha.

Nessa investigação, a geração de RCD foi estimada para os mesmos seis setores considerados no estudo da Franklin Associates (1998) e de Cochran et al. (2007): nova construção residencial, nova construção não residencial, demolição residencial, demolição não residencial, reabilitação residencial e reabilitação não residencial. Neste artigo são apresentados os indicadores calculados para a nova construção residencial. Os RCD gerados em obras públicas não foram inclusos na estimativa por não existir praticamente nenhum estudo que quantifique os resíduos produzidos nesse tipo de obras. Várias publicações notam a 
ausência de tais dados, destacando também que uma elevada percentagem dos RCD corresponde a solos de escavação, que quase nunca são inclusos nos estudos sobre RCD, por não serem considerados um grande problema ambiental (MYHRE, 2000; KARTAM et al., 2004; WEISLEDER; NASSERI, 2006; KOFOWOROLA; GHEEWALA, 2009).

A metodologia utilizada para estimar a geração de RCD ao nível da obra foi baseada nos procedimentos do estudo de Cochran et al. (2007), ou seja, foram usados dados de estudos anteriores sobre RCD existentes na literatura. Esses dados, recolhidos de investigações anteriores, foram determinados através da avaliação da composição dos resíduos gerados em obra. Somente foram inclusos estudos que mencionam a área associada à atividade em análise. Pelo fato de se pretender obter os valores da geração de RCD em quilogramas por metro quadrado, foi necessário converter os valores que se exibiam em metros cúbicos por metros quadrados usando as densidades dos materiais (Quadro 2).

Relativamente à composição dos resíduos, optouse por seguir as regras da LER por este ser um documento legislativo central no quadro jurídico europeu relativo à classificação de resíduos. De todos os fluxos de resíduos presentes na LER, apenas foram contabilizados aqueles que se incluem no capítulo 17 (resíduos de construção e demolição), por se assumir que esse grupo contribui efetivamente com maior peso para a quantificação global de resíduos na obra. Como mencionado, embora pertençam ao capítulo 17 da LER e representem uma parte significativa dos RCD gerados, os solos de escavação e lamas de dragagem foram excluídos desse levantamento. A quantidade produzida desses resíduos deve ser estimada com base em informação específica disponível no local da obra. Existem vários códigos relativos a resíduos contendo substâncias perigosas no capítulo 17. No entanto, neste estudo os resíduos perigosos foram todos inclusos no código $17 \quad 09 \quad 03$ (resíduos de construção e demolição contendo substâncias perigosas), por não existirem dados suficientes para os diferenciar. No Quadro 3, apresentam-se os resíduos que foram contabilizados, bem como o código LER no qual se incluem.

\begin{tabular}{l|c}
\hline \multicolumn{1}{c|}{ Material } & Densidade $\left(\mathbf{k g} / \mathbf{m}^{3}\right)$ \\
\hline Madeira & 178,0 \\
Gesso cartonado & 207,7 \\
Estuque (gesso) & 593,3 \\
Alvenaria (de tijolo) & 830,6 \\
Plásticos & 13,0 \\
Metais & 900,0 \\
Vidro & $2.500,0$ \\
Mistura RCD (geral) & 830,6 \\
\hline
\end{tabular}

Quadro 2 - Densidades dos materiais soltos consideradas para o cálculo da geração de RCD, em peso

Fonte: Coelho e Brito (2010), Hao, Tam e Hills (2007), Franklin Associates (1998) e Giancoli (1998).

\begin{tabular}{l|c}
\hline \multicolumn{1}{c|}{ Resíduos } & Código LER \\
\hline Betão & 170101 \\
Tijolos & 170102 \\
Ladrilhos, telhas e materiais cerâmicos & 170103 \\
Misturas de betão, tijolos, ladrilhos, telhas e materiais cerâmicos & 170107 \\
Betão, tijolos, ladrilhos, telhas e materiais cerâmicos & 1701 \\
Madeira & 170201 \\
Vidro & 170202 \\
Plástico & 170203 \\
Misturas betuminosas & 170302 \\
Mistura de metais & 170407 \\
Materiais de isolamento & 170604 \\
Materiais de construção à base de gesso & 170802 \\
Resíduos de construção e demolição contendo substâncias perigosas & 170903 \\
Mistura de resíduos de construção e demolição & 170904 \\
\hline
\end{tabular}

Quadro 3 - Resíduos contabilizados nesta pesquisa e código LER no qual se incluem

Fonte: Mália (2010).

122 Mália, M.; Brito, J. de; Bravo, M. 
A informação disponível sobre a geração de RCD é limitada (COCHRAN et al., 2007), por isso não foi possível produzir indicadores que diferenciassem as principais características dos edifícios (tais como o número de pisos e o tipo de fundações, estrutura e cobertura). Contudo, sabendo-se da importância do tipo de estrutura do edifício para a quantidade e composição dos resíduos gerados, procurou-se, sempre que possível, produzir indicadores que diferenciassem essa característica (embora a amostra de edifícios com estrutura metálica fosse demasiado pequena para ser tratada). Essa informação é também bastante heterogênea, o que conduziu à necessidade de se produzir um intervalo de valores para a geração de RCD, em vez de se tentar gerar um único valor. Essa solução foi também utilizada no estudo de Myhre (2000). Segundo Lage et al. (2010), a grande amplitude dos dados disponíveis pode ser atribuída a uma série de causas diferentes: falta de estudos fiáveis, diferenças de poder econômico, tamanho das cidades, diferentes práticas construtivas e diferenças relacionadas com o tipo predominante de população (rural ou urbana). Sabendo-se que os dados recolhidos são influenciados por diversos fatores, optou-se por atribuir um grau qualitativo de confiança aos indicadores desenvolvidos (Quadro 4), de modo a ter-se uma noção de seu nível de fiabilidade.

Para simplificar a utilização dos indicadores de RCD produzidos, foram utilizados os valores de geração em quilogramas por metro quadrado. Assim, apenas é necessário multiplicar os indicadores pela área bruta do edifício ou pela área de intervenção, no caso de trabalhos de reabilitação, para se obter a geração de RCD ao nível da obra.

\section{Indicadores de RCD propostos}

Em seguida, são apresentadas algumas considerações feitas, bem como os resultados obtidos para os indicadores de geração de RCD ao nível da obra (em nova construção), para edifícios residenciais.

Para a determinação dos indicadores de geração de RCD relativos a essa categoria, foram contabilizados 27 estudos. No Quadro 5, pode-se observar uma amostra dos dados recolhidos. Após uma análise cuidadosa, foram excluídos alguns outliers. Em seguida, para os materiais dos códigos LER presentes de forma significativa, procede-se à análise dos indicadores desenvolvidos para a nova construção residencial.

Na Figura 1, apresentam-se os dados concernentes ao código 170101 (betão). Como se vê no Quadro 6, a geração de resíduos de betão, para edifícios com a estrutura em madeira, situa-se entre 0,3 e $1,9 \mathrm{~kg} / \mathrm{m}^{2}$. Esse resultado foi obtido através da análise dos estudos que avaliam apenas a geração de resíduos de betão em edifícios com estrutura em madeira. Os dados relativos a edifícios com a estrutura em betão armado são menos concludentes, encontrando-se valores entre 2,5 e $4,5 \mathrm{~kg} / \mathrm{m}^{2}$ e entre 17,8 e $32,9 \mathrm{~kg} / \mathrm{m}^{2}$. Contudo, uma análise mais detalhada dos dados sugere que a estimativa entre 2,5 e $4,5 \mathrm{~kg} / \mathrm{m}^{2}$ resulta da constatação de que parte dos resíduos de betão se encontra misturada com outras frações de resíduos, tendo sido inclusos num código diferente (17 01 07 - misturas de betão, tijolos, ladrilhos, telhas e materiais cerâmicos). Conclui-se assim que, para edifícios com a estrutura em betão armado, a produção de resíduos de betão deve situar-se entre 17,8 e $32,9 \mathrm{~kg} / \mathrm{m}^{2}$. No entanto, caso se contabilize uma parte desses resíduos como fazendo parte do código 1701 07, esse valor pode ser inferior.

\begin{tabular}{|c|l|}
\hline $\begin{array}{c}\text { Grau de } \\
\text { confiança }\end{array}$ & \multicolumn{1}{c|}{ Descrição } \\
\hline Bom & $\begin{array}{l}\text { Contabilizaram-se muitos valores, apresentando-se estes homogêneos. } \\
\text { Valores bastante conclusivos. }\end{array}$ \\
\hline Razoável & $\begin{array}{l}\text { Contabilizaram-se muitos valores, apresentando-se estes heterogêneos. } \\
\text { Valores pouco conclusivos. }\end{array}$ \\
\hline Fraco & $\begin{array}{l}\text { Contabilizaram-se poucos valores. É possível, no entanto, retirar conclusões } \\
\text { deles. }\end{array}$ \\
\hline Mau & $\begin{array}{l}\text { Recolheu-se uma amostra muito reduzida de valores. Não é possível retirar } \\
\text { conclusões deles. }\end{array}$ \\
\hline
\end{tabular}

Quadro 4 - Escala utilizada na atribuição de um grau de confiança aos indicadores desenvolvidos Fonte: Mália (2010). 


\begin{tabular}{|c|c|c|c|c|c|c|}
\hline \multirow{12}{*}{$\begin{array}{l}\text { Geração de } \\
\text { RCD por } \\
\text { código LER } \\
\left(\mathbf{k g} / \mathbf{m}^{2}\right)\end{array}$} & 170904 & 9,60 & 6,19 & 0,87 & 0,87 & 20,99 \\
\hline & 170802 & 3,04 & 1,38 & 5,93 & 5,93 & 7,64 \\
\hline & 170604 & 1,20 & 0,21 & & & 3,32 \\
\hline & 170407 & 0,11 & 0,48 & 3,38 & 3,93 & 2,80 \\
\hline & 170203 & & & 0,14 & 0,15 & 2,94 \\
\hline & 170202 & 0,24 & 0,12 & & & \\
\hline & 170201 & 5,68 & 2,75 & 2,52 & 0,99 & 12,46 \\
\hline & 170107 & 6,50 & 19,11 & 96,92 & 96,92 & 38,65 \\
\hline & 170103 & & & & & 1,86 \\
\hline & 170102 & & & & & 29,24 \\
\hline & 170101 & & & 3,29 & 4,47 & 32,90 \\
\hline & Total & 29,36 & 30,77 & 114,26 & 114,47 & 168,05 \\
\hline \multicolumn{2}{|c|}{ Estudo } & \multicolumn{2}{|c|}{$\begin{array}{c}\text { Bergsdal, Bohne e } \\
\text { Bratterø (2007) }\end{array}$} & \multicolumn{2}{|c|}{$\begin{array}{c}\text { Mañà i Reixach, } \\
\text { Gonzàlez i Barroso } \\
\text { e Sagrera i Cuscó } \\
(2000)\end{array}$} & $\begin{array}{l}\text { BRE } \\
(2010)\end{array}$ \\
\hline \multicolumn{2}{|c|}{ País } & \multicolumn{2}{|c|}{ Noruega } & \multicolumn{2}{|c|}{ Espanha } & $\begin{array}{l}\text { Reino } \\
\text { Unido }\end{array}$ \\
\hline
\end{tabular}

Quadro 5 - Dados de geração de RCD em obra, determinados em estudos anteriores, na atividade de nova construção residencial ${ }^{*}$

Nota: *Foram ainda contabilizados dados do estudo de Myhre (2000), Metro Vancouver (2010), Kelly e Hanahoe (2009), Grimes (2005), Kofoworola e Gheewala (2009), Kharrufa (2007), Stenis (2005), Fatta et al. (2003), Wang e Zhao (2003), Solís-Guzman et al. (2009), Ortiz, Pasqualino e Castells (2010), Lage et al. (2010), Hsiao et al. (2002), Katz e Baum (2010), Giglio (2002), McGregor, Washburn e Palermini (1993), NAHB Research Center (1995), Cochran et al. (2007), Salinas (2002), Franklin Associates (1998), 3D/ International (1999), Lipsmeier e Günther (2002), Kartam et al. (2004) e Kibert (2002).

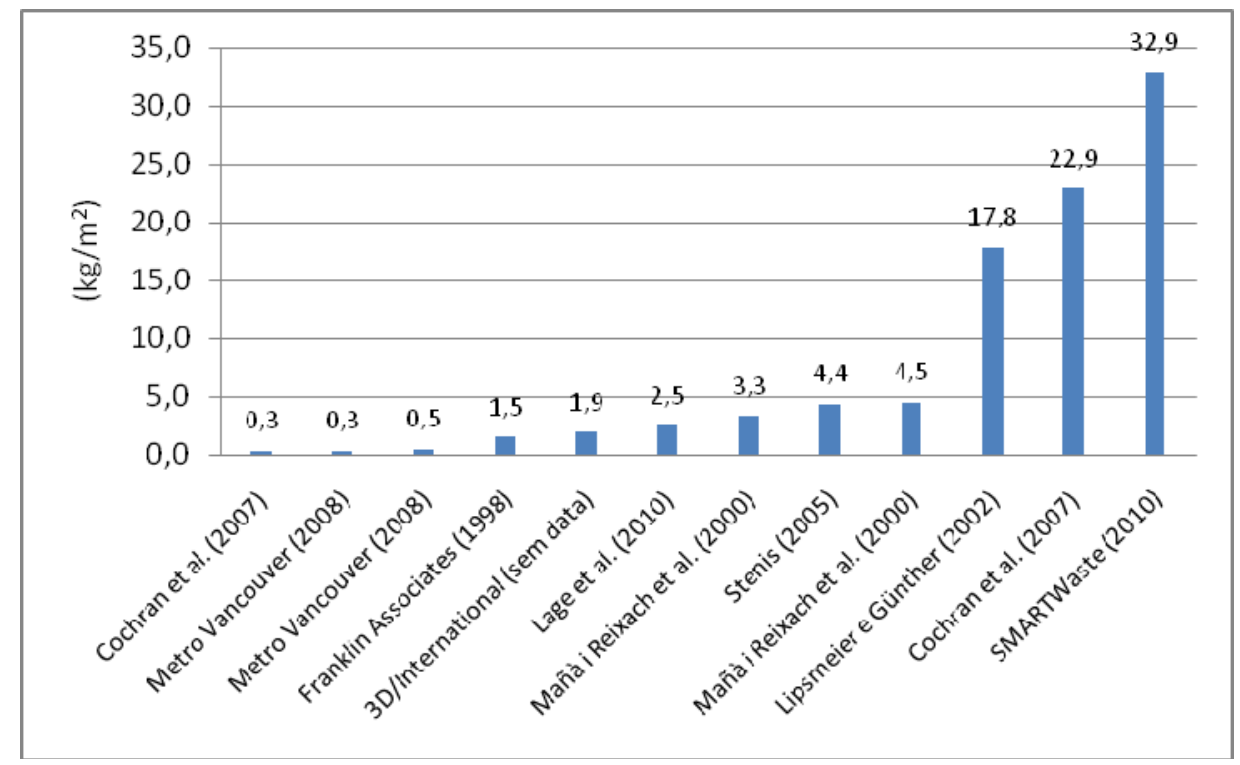

Figura 1 - Distribuição dos valores referentes ao código $170101\left(\mathrm{~kg} / \mathrm{m}^{2}\right)$

Fonte: Mália (2010).

Em relação aos valores referentes ao código 1701 02 (tijolos), não foram contabilizados muitos dados. Mesmo assim, foi possível distinguir duas tendências. Para edifícios com a estrutura em betão armado, a produção desse fluxo de resíduos situase entre 19,2 e 58,6 kg/m². Para edifícios com a estrutura em madeira, a geração de resíduos de tijolos situa-se entre 0,5 e $0,8 \mathrm{~kg} / \mathrm{m}^{2}$.

Os dados relativos aos resíduos de betão, tijolos, ladrilhos, telhas e materiais cerâmicos (subcapítulo 170103 da LER) apresentam, em alguns casos, apenas uma estimativa global da mistura desses resíduos. Nesta investigação, produziram-se 
indicadores para cada um desses fluxos de resíduos, mas, também, para sua totalidade. O desenvolvimento de um indicador para a geração total de resíduos do subcapítulo 1701 implica que a totalidade dos resíduos estimados para os diversos códigos desse subcapítulo situe-se dentro da estimativa aconselhada por esse indicador. Dessa forma, possibilita-se que as estimativas realizadas para cada fração sejam diferentes das desenvolvidas nesta investigação sem, no entanto, se comprometer a real dimensão da quantidade gerada desses resíduos. Assim, obtiveram-se duas estimativas distintas. Em edifícios com estrutura em betão armado, a geração total de RCD (associados ao subcapítulo 17 01) situa-se entre 40 e $102 \mathrm{~kg} / \mathrm{m}^{2}$. Em edifícios com estrutura em madeira, ela se situa entre 0 e $2 \mathrm{~kg} / \mathrm{m}^{2}$. Embora esse indicador global tenha como objetivo complementar os restantes indicadores desenvolvidos (associados ao subcapítulo 17 01), não foi obtido de forma totalmente dependente destes. Esse processo visou evitar que indicadores menos fiáveis colocassem em dúvida a geração real dessas frações de resíduos. Ainda assim, podese constatar que a estimativa desenvolvida acaba por ser semelhante à que resultaria do somatório dos indicadores produzidos para os vários códigos LER que compõem esse subcapítulo.

Os valores referentes ao código 170201 (madeira) apresentam certa homogeneidade, podendo-se estimar a geração desse fluxo de resíduos entre 2,5 e $17,9 \mathrm{~kg} / \mathrm{m}^{2}$ (Quadro 6). Contudo, após uma análise mais cuidadosa dos dados existentes, podem ser identificadas duas tendências distintas dentro desse intervalo. Os edifícios com a estrutura em madeira situam-se entre 5,6 e $17,9 \mathrm{~kg} / \mathrm{m}^{2}$, enquanto os edifícios com a estrutura em betão armado situam-se, principalmente, entre 2,5 e 6,4 $\mathrm{kg} / \mathrm{m}^{2}$. Para edifícios com a estrutura em betão armado, a geração de resíduos de madeira é condicionada pelo tipo de cofragem utilizado. Assim, pode-se esperar valores inferiores aos estimados caso não sejam utilizadas cofragens de madeira.

Não existem grandes discrepâncias nos dados referentes ao código $1708 \quad 02$ (materiais de construção à base de gesso), variando estes entre 2,4 e $7,6 \mathrm{~kg} / \mathrm{m}^{2}$. A geração de resíduos associados a esse código é condicionada pela maior ou menor utilização de revestimentos à base de gesso e placas de gesso cartonado. Da interpretação dos dados obtidos, pode-se concluir ainda que, para edifícios com a estrutura em betão armado, o intervalo deve situar-se entre 3,7 e $7,6 \mathrm{~kg} / \mathrm{m}^{2}$. Para edifícios com a estrutura em madeira, este se situa entre 2,4 e $7,2 \mathrm{~kg} / \mathrm{m} 2$.

No Quadro 6, pode-se ainda observar os indicadores de geração de RCD para os seguintes códigos LER: 170103 (ladrilhos, telhas e materiais cerâmicos), 170202 (vidro), 170203 (plástico), 170302 (misturas betuminosas), 1704 07 (mistura de metais), 170604 (materiais de isolamento), 170903 (RCD contendo substâncias perigosas). Como se pode observar, estes tipos de materiais existem em menores quantidades neste tipo de obra (nova construção residencial).

\begin{tabular}{|c|c|c|c|c|c|}
\hline \multirow{2}{*}{ Código LER } & \multicolumn{4}{|c|}{ Tipo de estrutura do edifício } & \multirow{2}{*}{$\begin{array}{c}\text { Grau de } \\
\text { confiança }\end{array}$} \\
\cline { 2 - 5 } & Não definido & Madeira & Betão armado & Alvenaria & Fraco \\
\hline 170101 & - & $0,3-1,9$ & $17,8-32,9$ & - & Fraco \\
\hline 170102 & - & $0,5-0,8$ & $19,2-58,6$ & - & Mau \\
\hline 170103 & - & - & $1,7-3,2$ & - & Razoável \\
\hline 1701 & - & $0-2$ & $40-102$ & - & Bom \\
\hline 170201 & - & $5,6-17,9$ & $2,5-6,4$ & - & Mau \\
\hline 170202 & $0,0-0,3$ & - & - & - & Fraco \\
\hline 170203 & $0,1-0,8$ & - & - & - & Mau \\
\hline 170302 & $0,4-2,6$ & - & - & - & Razoável \\
\hline 170407 & - & $0,1-0,9$ & $0,9-3,9$ & $0,2-2,6$ & Fraco \\
\hline 170604 & $0,1-1,2$ & - & - & - & Bom \\
\hline 170802 & - & $2,4-7,2$ & $3,7-7,6$ & - & Mau \\
\hline 170903 & $0,02-0,33$ & - & - & - & Bom \\
\hline Total & - & $\mathbf{1 0}-\mathbf{3 9}$ & $\mathbf{4 4 - 1 1 5}$ & - & \multirow{2}{*}{} \\
\hline
\end{tabular}

Quadro 6 - Indicadores de RCD $\left(\mathbf{k g} / \mathbf{m}^{2}\right)$

Fonte: Mália (2010). 
$\mathrm{Na}$ fase de acabamento, na qual os resíduos produzidos consistem de uma mistura de todos os materiais encontrados no local de construção (KATZ; BAUM, 2010), os resíduos acabam muitas vezes misturados. Assim, desenvolveu-se uma estimativa para a geração global de RCD na obra, de modo a que os indicadores desenvolvidos nesta pesquisa possam considerar eventuais misturas de resíduos. O desenvolvimento de um indicador para a geração total de RCD implica que a totalidade dos resíduos estimados para os diversos códigos LER se situe dentro da estimativa aconselhada por este indicador. Os valores relativos à geração total de RCD para a nova construção residencial podem ser vistos na Figura 2.

Através destes dados, verificou-se que a geração total de RCD, para edifícios com a estrutura em madeira, se situa entre 10 e $39 \mathrm{~kg} / \mathrm{m} 2$ (Quadro 6). Para edifícios com a estrutura em betão armado, esta situa-se entre 44 e $115 \mathrm{~kg} / \mathrm{m} 2$. Optou-se pelo valor de $115 \mathrm{~kg} / \mathrm{m} 2$ para definir o limite máximo deste intervalo pois considerara-se pouco fiáveis os valores largamente superiores a $115 \mathrm{~kg} / \mathrm{m} 2$. Os dados de Kharrufa (2007) sugerem um elevado desperdício de materiais durante o processo construtivo, resultado das limitadas políticas de gestão de RCD no Iraque. Por sua vez, os dados de Hsiao et al. (2002) e de Ortiz, Pasqualino e Castells (2010) resultam do somatório de valores que foram considerados outliers. Embora este indicador global tenha como finalidade complementar os restantes indicadores desenvolvidos, não foi obtido de forma totalmente dependente destes. Assim, pretende-se evitar que indicadores com um baixo grau de fiabilidade possam adulterar a quantidade real de RCD produzidos em obra. Pode-se constatar que a estimativa desenvolvida acaba por ser semelhante à que resultaria do somatório dos diversos indicadores produzidos para a categoria em análise.

No Quadro 6, apresenta-se ainda o grau de confiança atribuído a cada um dos indicadores desenvolvidos para a nova construção residencial. O grau de confiança conferido a cada indicador depende, essencialmente, da quantidade de dados recolhidos e das conclusões possíveis de retirar destes. No Quadro 6, encontram-se realçados os indicadores que englobam vários códigos LER, para uma melhor compreensão.

\section{Conclusões}

Nesta investigação, efetuou-se uma pesquisa sobre a legislação e as estratégias seguidas em relação aos RCD, na União Europeia e no Brasil. Por meio dessa análise, foi possível identificar os aspectos positivos e negativos dos diversos documentos.

Foi possível verificar a importância da implementação de uma elevada taxa de deposição de RCD em aterro. Por exemplo, a taxa que atualmente é praticada em Portugal não promove a reciclagem de RCD, tornando mais rentável sua deposição do que sua reciclagem. A alternativa à taxação de deposição de RCD em aterro passa pela interdição da deposição em aterro de RCD recicláveis. Essa foi a medida adoptada pela Alemanha, também com grande sucesso.

Nesta investigação, produziram-se indicadores que fornecem à indústria da construção um conjunto de valores inovadores que podem ser utilizados como referência para quantificar, de forma simples e consistente, os RCD gerados ao nível da obra (em nova construção). Os indicadores podem ser utilizados para determinar as dimensões dos contentores e a frequência com que estes devem ser utilizados. O planejamento antecipado dessas ações possibilita que os resíduos sejam adequadamente tratados, impulsionando seu reaproveitamento.

A metodologia utilizada para desenvolver os indicadores baseou-se num levantamento exaustivo de dados de estudos anteriores. Foram também definidas diversas considerações, como complemento aos dados recolhidos. Verificou-se que foi possível obterem-se bastantes dados para a atividade de nova construção residencial, pois esse tipo de trabalho é amplamente monitorado e estudado.

De acordo com os indicadores desenvolvidos, conclui-se que a constituição média dos resíduos gerados em obra é composta maioritariamente de resíduos de betão e materiais cerâmicos. Esse resultado está de acordo com os resultados de outros estudos, em que se afirma que estes representam cerca de $80 \%$ do total de RCD gerados (quando não são contabilizados os solos de escavação). Dos restantes materiais, destacamse os resíduos de madeira e os materiais à base de gesso, gerados a partir de desperdícios associados à cofragem e à colocação de revestimentos respectivamente. 


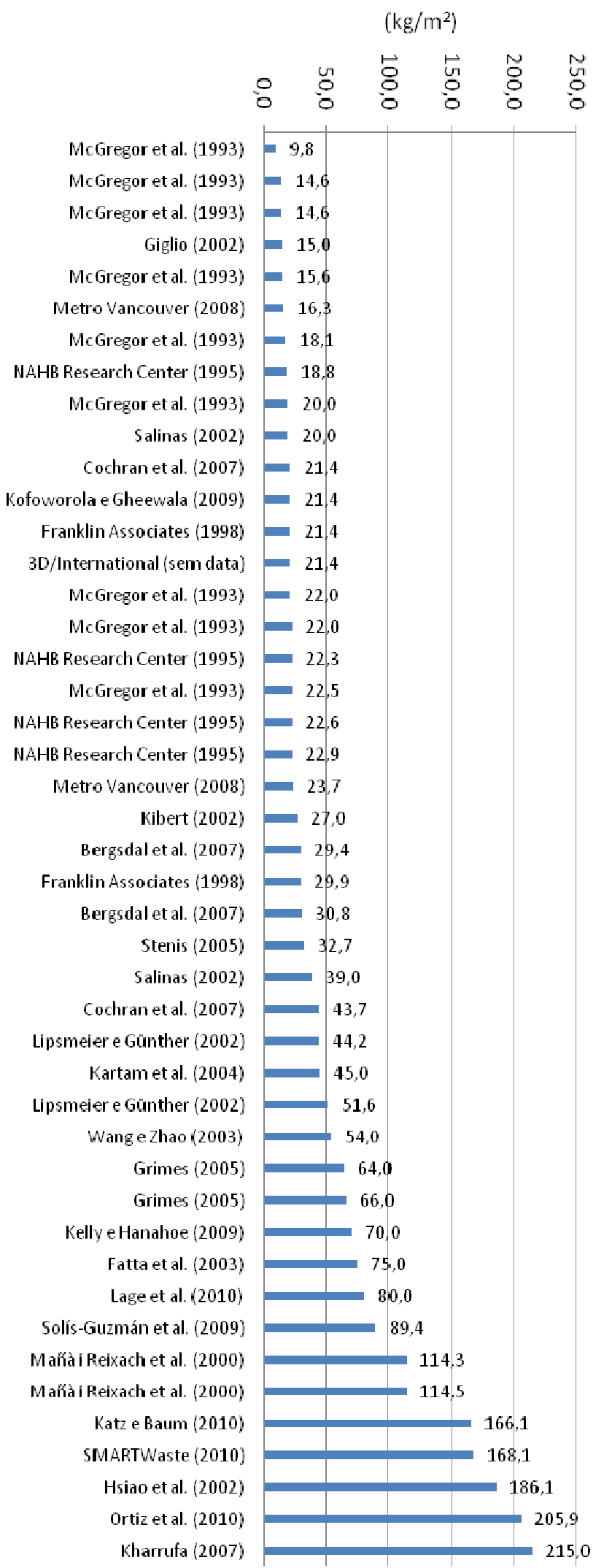

Figura 2 - Distribuição dos valores referentes à geração total de $\operatorname{RCD}\left(\mathbf{k g} / \mathrm{m}^{2}\right)$

Fonte: Mália (2010). 


\section{Referências}

3D/INTERNATIONAL. Construction and Demolition Waste Management Pocket Guide. HQ Air Force Center for Environmental Excellence. USA, 1999.

ANGULO, S. Variabilidade de Agregados Graúdos de Resíduos de Construção e Demolição Reciclados. 172 f. São Paulo, 2000. Dissertação (Mestrado em Engenharia Civil) Escola Politécnica, Universidade de São Paulo, São Paulo, 2000.

ASSOCIAÇÃO BRASILEIRA DE NORMAS TÉCNICAS. NBR 15112: diretrizes para projeto, implantação e operação de áreas de triagem e transbordo. Rio de Janeiro, 2004a.

ASSOCIAÇÃO BRASILEIRA DE NORMAS TÉCNICAS. NBR 15113: diretrizes para projeto, implantação e operação de aterros. Rio de Janeiro, 2004b.

\section{ASSOCIAÇÃO BRASILEIRA DE NORMAS}

TÉCNICAS. NBR 15114: diretrizes para projeto, implantação e operação de áreas de reciclagem. Rio de Janeiro, 2004c.

\section{ASSOCIAÇÃO BRASILEIRA DE NORMAS} TÉCNICAS. NBR 15115: procedimentos para execução de camadas de pavimentação utilizando agregados reciclados de resíduos da construção. Rio de Janeiro, 2004d.

\section{ASSOCIAÇÃO BRASILEIRA DE NORMAS}

TÉCNICAS. NBR 15116: requisitos para utilização em pavimentos e preparo de concreto sem função estrutural com agregados reciclados de resíduos da construção. Rio de Janeiro, 2004e.

BERGSDAL, H.; BOHNE, R.A.; BRATTEBØ, H. Projection of Construction and Demolition Waste in Norway. Journal of Industrial Ecology, v. 11, n. 3, p. 27-39, 2007.

BOSSINK, B. A. G.; BROUWERS, H. J. H. Construction Waste: quantification and source evaluation. Journal of Construction Engineering and Management, v. 122, n. 1, p. 55-60, 1996.

BRASIL. Conselho Nacional do Meio Ambiente. Resolução n. ${ }^{\circ}$ 237, de 19 de dezembro de 1997, procedimentos e critérios utilizados no licenciamento ambiental. Diário Oficial da União, 22 de dezembro de1997.

BRASIL. Conselho Nacional do Meio Ambiente. Resolução n. ${ }^{0}$ 307, de 02 de julho de 2002, que estabelece diretrizes, critérios e procedimentos para a gestão dos resíduos da construção civil. Diário Oficial da União, 05 de julho de 2002.
BRITISH RESEARCH ESTABLISHMENT. SMARTWaste Plan. Disponível em: $<$ http://www.smartwaste.co.uk>. Acesso em: 22 set. 2010.

COCHRAN, K. et al. Estimation of Regional Building-Related C\&D Debris Generation and Composition: case study for Florida, US. Journal of Waste Management, v. 27, n. 7, p. 921-931, 2007.

COELHO, A.; BRITO, J. de. Análise da Viabilidade de Implantação de Centrais de Reciclagem de Resíduos de Construção e Demolição em Portugal: parte 1, estimativa da geração de resíduos de construção e demolição. Lisboa, Instituto Superior Técnico, 2010. Relatório ICIST, DTC n. ${ }^{\circ}$ 04/2010.

COSTA, U.; URSELLA, P. Construction and Demolition Waste Recycling in Italy. In: INTERNATIONAL CONFERENCE ON THE ENVIRONMENTAL AND TECHNICAL IMPLICATIONS OF CONSTRUCTION, 5.; ALTERNATIVE MATERIALS "WASCON 2003”, San Sebastián, Spain, 2003. Proceedings... San Sebastián, Spain, 2003. p. 231-239.

EUROSTAT, ENVIRONMENT AND ENERGY. Generation and Treatment of Waste. Disponível em: <http://ec.europa.eu/eurostat/>. Acesso em: 25 set. 2010.

FATTA, D. et al. Generation and Management of Construction and Demolition Waste in Greece: an existing challenge. Journal of Resources, Conservation and Recycling, v. 40, n. 1, p. 8191, 2003.

FRANKLIN ASSOCIATES. Characterization of Building-related Construction and Demolition Debris in the United States. US Environmental Protection Agency, Municipal and Industrial Solid Waste Division, Office of Solid Waste. Report No. EPA530-R-98-010, Prairie Village, Kansas, United States of America, 1998.

GIANCOLI, D. C. Physics, Principles with Applications. $5^{\text {th }}$ ed. New Jersey: Prentice Hall, 1998.

GIGLIO, F. Controlling Environmental Impacts in the Dismantling Phase. In: CIB CONFERENCE DESIGN FOR DECONSTRUCTION AND MATERIALS REUSE, Karlsruhe, Germany, 2002. Proceedings... Karlsruhe, Germany, 2002. 
GRIMES, D. The Assessment of Construction and Demolition Wastes Arising on Selected Case Study Construction Projects in the Galway Region. 2005. M.Sc. (Thesis in Construction Management) - Department of Building and Civil Engineering, Galway-Mayo Institute of Technology, Galway, Ireland, 2005.

HAO, J. L.; TAM, V. W. Y.; HILLS, M. J. The Construction Waste Disposal Charging Scheme in Hong Kong. In: INTERNATIONAL

CONFERENCE ON CONSTRUCTION IN THE $21^{\text {ST }}$ CENTURY, 5., Gold Coast, Australia, 2007. Proceedings... Gold Coast, Australia, 2007.

HSIAO, Y. et al. Modeling Materials Flow of Waste Concrete from Construction and Demolition Wastes in Taiwan. Journal of Resources Policy, v. 28, n. 1/2, p. 39-47, 2002.

KARTAM, N. et al. Environmental Management of Construction and Demolition Waste in Kuwait. Journal of Waste Management, v. 24, n. 10, p. 1049-1059, 2004.

KATZ, A.; BAUM, H. A Novel Methodology to Estimate the Evolution of Construction Waste in Construction Site. Journal of Waste

Management, v. 31, n. 2, p. 353-358, 2010.

KELLY, M.; HANAHOE, J. Development of an Audit Methodology to Generate Construction Waste Production Indicators for the Irish Construction Industry. Ireland: Environment Protection Agency, 2009. STRIVE Report.

KHARRUFA, S. Reduction of Building Waste in Baghdad Iraq. Journal of Building and Environment, v. 42, n. 5, p. 2053-2061, 2007.

KIBERT, C. J. Deconstruction's Role in an Ecology of Construction. In: CHINI, A.; SCHULTMANN, F. (Eds.). Design for Deconstruction and Materials Reuse. Amsterdam: International Council for Research and Innovation in Building Construction, 2002. CIB Publication 272.

KOFOWOROLA, O. F.; GHEEWALA, S. H. Estimation of Construction Waste Generation and Management in Thailand. Journal of Integrated Waste Management, v. 29, n. 2, p. 731-738, 2009.

LAGE, I. M. et al. Estimation of the Annual Production and Composition of C\&D Debris in Galicia (Spain). Journal of Waste Management, v. 30, n. 4, p. 636-645, 2010.

LIPSMEIER, K.; GÜNTHER, M. Manual Europeu de Resíduos da Construção de Edifícios. Minho: Institute for Waste Management and Contaminated Sites Treatment of Dresden University of Technology, 2002. v. 1.
LISBOA. Decreto-Lei n. ${ }^{\circ}$ 488: resíduos sólidos. Diário da República, 1985.

LISBOA. Decreto-Lei n. ${ }^{\circ}$ 310: gestão de resíduos. Diário da República, 1995.

LISBOA. Decreto-Lei n. ${ }^{\circ}$ 239: gestão de resíduos. Diário da República, 1997.

LISBOA. Decreto-lei n. ${ }^{\circ}$ 178: gestão de resíduos. Diário da República, 2006.

LISBOA. Decreto-Lei n. o 46: gestão de resíduos de construção e demolição. Diário da República, 2008.

MÁLIA, M. Indicadores de Resíduos de Construção e Demolição. Lisboa, 2010. Dissertação (Mestrado em Engenharia Civil) Instituto Superior Técnico, Lisboa, 2010.

MAÑÀ I REIXACH, F.; GONZÀLEZ I BARROSO, J.; SAGRERA I CUSCÓ, A. Plan de Gestión de Residuos en las Obras de Construcción y Demolición. Catalunya, España: Dirección General de Medio Ambiente, Institut de Tecnología de la Construcció de Catalunya, 2000.

MCGREGOR, M.; WASHBURN, H.;

PALERMINI, D. Characterization of

Construction Site Waste. Portland, Oregon: METRO Solid Waste Department, 1993.

METRO VANCOUVER. Demolition, Land Clearing and Construction Waste Management Toolkit. Disponível em:

<http://www.metrovancouver.org>. Acesso em: 22 set. 2010.

MINISTÉRIO DAS CIDADES. Panorama dos Resíduos de Construção e demolição (RCD) no Brasil. Brasília, DF: Secretaria Nacional de Saneamento Ambiental, 2005.

MINISTERIO DE MEDIO AMBIENTE Y MEDIO RURAL Y MARINO. Plan Nacional Integrado de Residuos para el Período 20082015. Disponível em: <http://www.icespain.org.uk/about/index.aspx/>. Acesso em: 25 ago. 2010.

MONTECINOS, W.; HOLDA, A. Construction and Demolition Waste Management in Denmark. 2006. Disponível em: $<$ http://www.cowam-project.org/cms/>. Acesso em: 25 ago. 2010.

MYHRE, L. The State of Deconstruction in Norway. In: KIBERT, C. J.; CHINI, A. (Eds.). Overview of Deconstruction in Selected Countries. Amsterdam: International Council for Research and Innovation in Building Construction, 2000. p. 144-157. CIB Publication 252. 
NAHB RESEARCH CENTER, INC. Residential Construction Waste Management

Demonstration and Evaluation. US

Environmental Protection Agency, Office of Solid Waste, Task 1 Report, 1995.

ORTIZ, O.; PASQUALINO, J. C.; CASTELLS, F. Environmental Performance of Construction Waste: comparing three scenarios from a case study in Catalonia, Spain. Journal of Waste Management, v. 30, n. 4, p. 646-654, 2010.

PEREIRA, L. C. H. Reciclagem de Resíduos de Construção e Demolição: aplicação à zona norte de Portugal. Guimarães, 2002. Dissertação (Mestrado em Engenharia Civil) - Escola de Engenharia, Universidade do Minho, Guimarães, 2002.

PINTO, T. P. Metodologia para a Gestão Diferenciada de Resíduos Sólidos da Construção Urbana. São Paulo, 1999. Tese (Doutoramento em Engenharia Civil) - Escola Politécnica, Universidade de São Paulo, São Paulo, 1999.

PINTO, T. P.; AGOPAYAN, V. Construction Wastes as Raw Material for Low-cost Construction Products. In: INTERNATIONAL CONFERENCE CIB TG16, 1., Gainesville, 1994. Proceedings... Gainesville, 1994.

SALINAS, L. A. Gestão de Resíduos de Construção e Demolição: contributo para a avaliação e gestão municipal dos resíduos de construção em Portugal. Coimbra, 2002.

Dissertação (Mestrado em Construção) - Escola de Engenharia, Universidade de Coimbra, Coimbra, 2002.
SÓLIS-GUZMÁN, J. et al. A Spanish Model for Quantification and Management of Construction Waste. Journal of Integrated Waste Management, v. 29, n. 9, p. 2542-2548, 2009.

STENIS, J. Construction Waste Management Based on Industrial Management Models: a Swedish case study. Journal of Waste Management \& Research, v. 23, n. 1, p. 13-19, 2005.

WANG, L.; ZHAO, Y. Management and Resourcelization of Construction Waste. Beijing, China: Chemical Industry Press, 2003.

WASTE CENTRE DENMARK. Waste from Buildings and Construction Activities.

Disponível em: <http://www.wasteinfo.dk>. Acesso em: 20 set. 2010.

WEISLEDER, S.; NASSERI, D. Construction and Demolition Waste Management in Germany. 2006. Disponível em:

$<$ http://www.cowam-project.org/cms/>. Acesso em: 25 ago. 2010.

WIENS, I.; HAMADA, J. Gerenciamento de Resíduos da Construção Civil: uma introdução à legislação e implantação. In: SIMPÓSIO DE ENGENHARIA DE PRODUÇÃO DA UNESP, 13., Bauru, 2006. Anais... Bauru, 2006.

\section{Agradecimentos}

São devidos agradecimentos à FCT (Fundação para a Ciência e Tecnologia).

Revista Ambiente Construído

Associação Nacional de Tecnologia do Ambiente Construído

Av. Osvaldo Aranha, 99 - 3o andar, Centro Porto Alegre - RS - Brasil

CEP 90035-190

Telefone: +55 (51) 3308-4084

Fax: +55 (51) 3308-4054

www. seer. ufrgs. br/ ambienteconstruido

E-mail: ambienteconstruido@ufrgs.br 